\title{
Correction to: TP63 links chromatin remodeling and enhancer reprogramming to epidermal differentiation and squamous cell carcinoma development
}

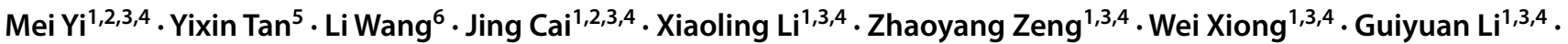 \\ Xiayu $\mathrm{Li}^{3} \cdot$ Pingqing $\operatorname{Tan}^{1,7} \cdot$ Bo Xiang ${ }^{1,3,4}$
}

Accepted: 15 October 2021 / Published online: 2 November 2021

(c) Springer Nature Switzerland AG 2021

\section{Correction to: \\ Cellular and Molecular Life Sciences (2020) 77:4325-4346 \\ https://doi.org/10.1007/s00018-020-03539-2}

The authors of the article would like to update the affiliation of the prof. Mei Yi.

The updated affiliations is follow:

${ }^{1}$ NHC Key Laboratory of Carcinogenesis, Hunan Cancer Hospital and the Affiliated Cancer Hospital of Xiangya School of Medicine, Central South University, Changsha 410,013, Hunan, China

The original article can be found online at https://doi.org/10.1007/ s00018-020-03539-2.

Xiayu Li

lixiayu@csu.edu.cn

Pingqing Tan

tanpingqing@hnszlyy.com

Bo Xiang

xiangbolin@csu.edu.cn

Mei Yi

yi_mei@csu.edu.cn

Yixin Tan

tyx0711@csu.edu.cn

Li Wang

li-wang@csu.edu.cn

Jing Cai

caijing@csu.edu.cn

Xiaoling Li

lixiaoling@csu.edu.cn

Zhaoyang Zeng

zengzhaoyang@csu.edu.cn

Wei Xiong

xiongwei@csu.edu.cn

Guiyuan $\mathrm{Li}$

lgy@csu.edu.cn
${ }^{2}$ Department of Dermatology, Xiangya Hospital, Central South University, Changsha 410,008, Hunan, China

${ }^{3}$ Hunan Key Laboratory of Nonresolving Infammation and Cancer, The Third Xiangya Hospital, Central South University, Changsha 410,013, Hunan, China

${ }^{4}$ The Key Laboratory of Carcinogenesis and Cancer Invasion of the Chinese Ministry of Education, Cancer Research Institute and School of Basic Medical Sciences, Central South University, Changsha 410,078, Hunan, China

Publisher's Note Springer Nature remains neutral with regard to jurisdictional claims in published maps and institutional affiliations.

1 NHC Key Laboratory of Carcinogenesis, Hunan Cancer Hospital and the Affiliated Cancer Hospital of Xiangya School of Medicine, Central South University, Changsha 410013, Hunan, China

2 Department of Dermatology, Xiangya Hospital, Central South University, Changsha 410008, Hunan, China

3 Hunan Key Laboratory of Nonresolving Infammation and Cancer, The Third Xiangya Hospital, Central South University, Changsha 410013, Hunan, China

4 The Key Laboratory of Carcinogenesis and Cancer Invasion of the Chinese Ministry of Education, Cancer Research Institute and School of Basic Medical Sciences, Central South University, Changsha 410078, Hunan, China

5 Department of Dermatology, The Second Xiangya Hospital, The Central South University, Changsha 410011, Hunan, China

6 Department of Thoracic Surgery, The Second Xiangya Hospital, Central South University, Changsha 410011, Hunan, China

7 Department of Head and Neck Surgery, Hunan Provincial Cancer Hospital and Cancer Hospital Affiliated to Xiangya Medical School, Central South University, Changsha 410013, Hunan, China 